\title{
COVID-19: A Mechanistic Approach to Understand the Detrimental Consequences for Vaccine Development and Perspectives
}

\author{
Chander Amgoth ${ }^{1, *}$, Tirupathi Malavath ${ }^{2}$, Guping Tang ${ }^{1, *}$ \\ ${ }^{1}$ Department of Chemistry, Zhejiang University, Hangzhou-310028, China \\ ${ }^{2}$ Department of Biochemistry and Molecular Biology, Tel Aviv University, Israel \\ *Corresponding author: E-mail: chander@uohyd.ac.in (C.A.); tangguping@zju.edu.cn (G.T.); Tel.: (+91) 9908812120 \\ DOI: 10.5185/amlett.2020.091551
}

Herein, the mechanistic approach to understanding the characteristic properties of the deadliest virus (COVID-19) has been corroborated. The nano-sized (160-200 nm, in diameter) virus along with the crucial role of spikes (length $\sim 10 \mathrm{~nm}$ ) has been deliberated. The distorted protein envelope followed by the aminoacid sequence mismatching lead to replicate the virus. Fragmented ssRNA further make duplicate template copies through the mutations for net-work entanglement. Mutations lead to an increase in population density of virus uncountable. Drastic increase in the virus shows an adverse effect on the immune system. The inflamed sacs of lungs will not allow the exchange of gases and ultimately lead to pneumonia/SARS-CoV2 (severe acute respiratory syndrome of coronavirus). The glycoprotein of spike spindles fuses the surface of lung cells through the binding affinity and slowdown the activity of antibodies. Inflammation caused because of the replicated complex of virus blocks the passage of gases $\mathrm{O}_{2}, \mathrm{CO}_{2}$ which lead to severe cough and respiratory problems. This unusual phenomenon of pneumonia leads to death. Further, massive computing is needed to understand the exact detrimental consequences of COVID-19 followed by the drug and vaccine development.

\section{Introduction}

As per the WHO, COVID-19 is renamed from Corona Virus Disease-2019 (CO-Corona, VI-Virus and D-stands for disease followed by the year 2019) [1]. As per the literature, many viruses are existing such as TMV (Tobacco Mosaic Virus), CPMV (Cowpea Mosaic Virus), Poliovirus, H1N1 (Swine flu), AIDS (Acquired Immunodeficiency Syndrome), Chickenpox, smallpox, Zika, Influenza, Nipah, Rabies, Ebola, SARS (Severe Acute Respiratory Syndrome), MARS (Middle East Respiratory Syndrome), Measles, common cold, herpes etc. [2]. Virology deals with the viruses to categorize and characterize. Viruses are so small in size but plays a crucial role in the evolution or origin of life $[\mathbf{1 , 2}]$. All viruses are not pathogenic as we think; there are many viruses that are benign to human beings. Few viruses cause the diseases and the majority of the viruses are just parasites to many lives [3]. Viruses are contentious and anticipated to predate the cells. However, viruses are the most abundant agents on the planet earth. Every environment in and around us is surrounded by the varieties of viruses [4]. The word virus itself is controversial when it comes to discussing the living or nonliving. Very few studies say about it is a living agent but the majority of studies says it is a non-living agent with a combination of just genetic materials encoded with protein envelope [5]. The disorders or mismatch in such encoded protein capsid lead to mutate or replicate based on environmental conditions. The interior of viruses is packed/filled with genetic materials (RNA/DNA) in the form of helix strands $[\mathbf{3 , 6}]$. The packed protein layer considered as a capsid. Basically, proteins made of aminoacids and surrounded by envelope. Virions are in nanometre size in the range of 160-180 $\mathrm{nm}$ (in diameter). The size, shape and morphology of viruses can be seen under the ultra-high resolution microscopes such as TEM (Transmission Electron Microscope), FE-SEM (Field Emission Scanning Electron Microscope) etc. Researchers and scientists refer to these viruses as quasi-life materials/particles $[\mathbf{5 , 6}$. Their main aim is to invade the cells of host material by releasing the genetic material to hijack and disrupt the daily activities of the normal metabolic cells. These altering capacities of the virus are because of disordered and mismatched protein envelop/amino-acid sequences [7]. This disrupted capsid allows the genetic material to replicate into make several copies themselves and ultimately mutations will occur. Simply, these viruses are termed as parasites of genetic material. Some of the viruses are simple to formulate their chemical formula but for some viruses, it's very difficult to formulate and characterize. In an aspect, these viruses are merely non-living bags of chemicals or genetic material [8]. On the other side, these viruses with genetic material are called biological entities which are capable of reproducing or replicating themselves to a large scale complex of giant 


\section{Advanced Materials Letters www.vbripress.com/aml}

molecule. However, there is a need to do cloud computing to understand the exact reason for the detrimental consequences of the virus [5-8]. Remarkably, a step by step mechanistic approach has been described in the following sections. The speculations and hypothesis about the virus has to be validated in a mechanistic approach which further helps in drug design and vaccine development.

\section{Results and discussion}

\section{Size, shape and morphology}

Understanding of characteristic physical properties of virus is a primary task to go for the design and development of drugs or vaccines. However, 65-70 percentages of these COVID-19 viruses have the genome/genetic material which is made-up of RNA and rest is of DNA genome [8]. This RNA and DNA genome has been encoded with different types of structural protein enveloped such as capsids, components of double envelopes and spike proteins. The COVID-19 viruses have these spike proteins which can be used to break/penetrate the host cells. The average size/length of these spikes is in the range of $10-12 \mathrm{~nm}[\mathbf{7}, 8]$.
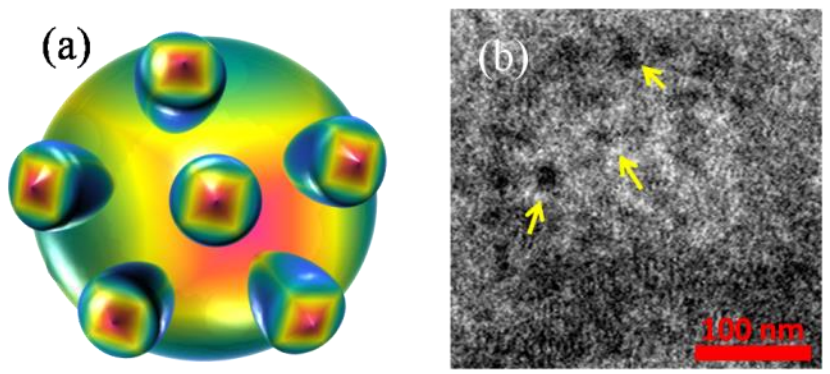

Fig. 1. Schematic (a) pictorial representation of morphology of virus and image (b) corresponds to the TEM of the virus morphology with surface distributed spikes (Yellow arrows).

The oral size of the virus is in the range of 160 to 200 $\mathrm{nm}$ (in diameter). These viruses are covered with the protein membrane which is made-up of various aminoacids. The central dogma of the virus has been internalized with the genetic material/ssRNA [9]. Fig. 1(a) schematic corresponds to the appearance of the COVID-19 virus. Similarly, Fig. 1(b) TEM (Transmission Electron Microscope) image corroborates the nano-sized virus with its spikes (highlighted with yellow arrows) distributed over the surface of the central dogma of the virus [10].

\section{Spikes and role}

Intriguing the virus interior and its detrimental consequences has been speculated in a mechanistic method to explore for better understanding. These understandings merely help to design a drug or development of a vaccine. Interestingly, spikes have the capability to penetrate the cell membrane through the net-work entanglement [7-10]. The affinity of spikes with the humidified atmosphere/cell surface helps virus to internalize inside the cell. Intensive research is needed to explore the hypothesis behind the detrimental consequences and adverse effect of virus on the respiratory system. As per its adverse effects, it has been speculated that this nanoscale non-living agent (foreign object) have the affinity with humidified lung cells which mutates drastically [11]. Moreover, fibrils/spindles of spikes act as a ligand to form a net-work with antibodies/ACE2 (angiotensin covering enzyme) and ARB2 (angiotensin receptor blockers). Sudden entanglement of viruses with receptor and antibodies lead to inactive and antibodies does not invade on the foreign agents (virus). Fig. 2(a,b) deliberates about the spikes and their internal spindles to entangle for net-work formulation. However, surface oriented spikes play crucial role to insert virus into the lung cells [9-11]. To understand in detail about the role of glycoprotein based spikes needs massive computing about its extreme domain complexation with antibodies.

\section{Central dogma of virus}

Fig. 2(c,d) corroborates the central dogma and internally oriented genetic material followed by the fragmentation of template copies of ssRNA through the replication. However, this COVID-19 has been referred as SARS$\mathrm{CoV} 2$ based on its detrimental consequences and symptoms [12]. It has been observed that the protein sequence of COVID-19 and SARS-CoV2 are paralogous which are the ancestral gene followed by the duplication for further evolution.

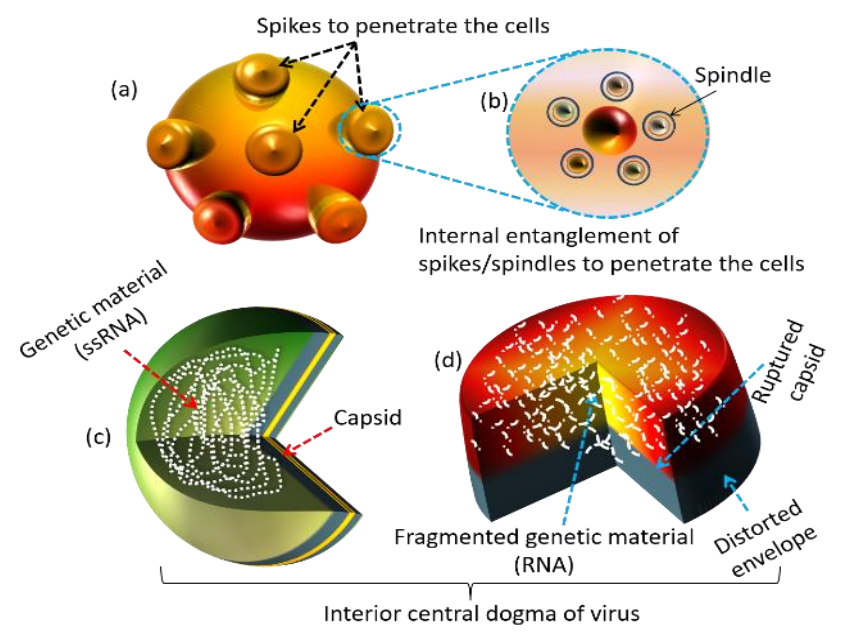

Fig. 2. Schematic (a) corresponds to the morphology of COVID-19 virus with surface distributed spikes, (b) interior of spikes with cell penetrating spindles and (c, d) corresponds to the central dogma of virus consist of genetic material and capsid of the virus.

Retrospectively, when these COVID-19 present in various species, it will be diverged after the duplication from its parent genetic material. From Fig. 2(c,d), we can see the disintegrated genetic material which further act as an individual virus and produces the more and more colonies of the same. This kind of effervescent activity of virus leads to the deactivation of antibodies and finally makes them to become neutral [13]. However, the immune system stimulates the antibodies to fight with foreign/unwanted agents through the macrophages development $[\mathbf{1 0}, \mathbf{1 3}]$. Typically, it is a phagocyte white 


\section{Advanced Materials Letters www.vbripress.com/aml}

blood cell found in a stationary form. Interestingly, this has the ability to locate and eat-up/destruct the foreign objects like bacteria, fungi, virus and other parasites. The main role of the immune system or antibodies is to release strong acids to kill the foreign unwanted agents like viruses. But due to drastic mutations, virus has the capability to form an aggregate complex structure which cannot be easily killed or destructed by the released acids. It is the very specific characteristic property of the COVID-19 virus which reverse invades against the immune system (antibodies). At this stage natural killer (T-cells) will also become neutral and cannot show much activity on virus [11-14].

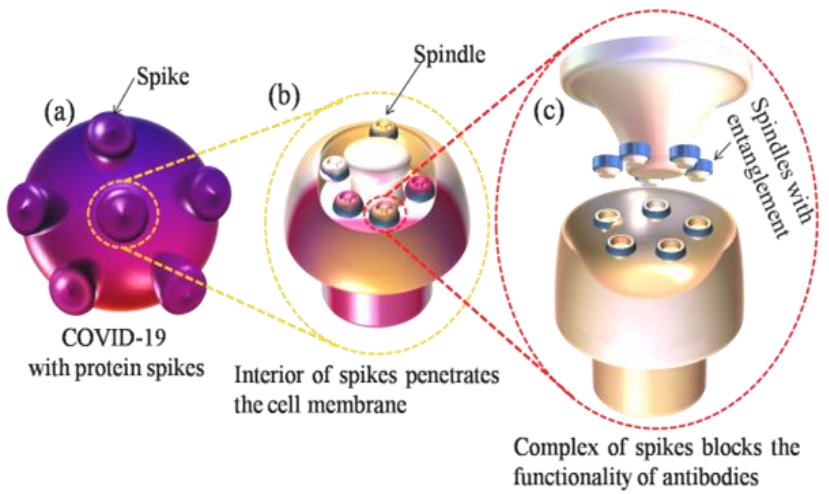

Fig. 3. Schematic (a) corresponds to the morphology of the COVID-19 virus with surface distributed spikes, (b) interior of spikes arranged with cell penetrating spindles and (c) corroborates the spikes with entanglement to penetrate the cell membrane and internalization at humidified condition of lungs.
Further, Fig. 3 corroborates the interior arrangement of spikes with entanglement. The complex of spindles blocks the activity of antibodies through the net-work formation with lung cells [14]. Typically, these spikes are glycoprotein based peplomers on the surface of capsid of virus. These protrusions will act as drilling agents with the help of fibrils and spindles and binds to the receptors of the hostile cell for complexation [15]. However, these glycosylated triangular spike proteins are identified as the $\mathrm{ACE} / \mathrm{ARB}$ receptor and shows activity through the ligand binding evolutionary intermediate mutagenesis in COVID19. Initially, the coronavirus spike protein shows a kind of affinity with the cells and starts infecting the cell surface to enter into the cell for more severe damage $[\mathbf{1 4 , 1 5}$. Slowly, the virus can fuse with the cellular membrane fluids and releases the genetic material/RNA inside the cell then actual duplication of virus template copies will start to enhance its count. Interestingly, these spike protein (glycoprotein) is the strongest and sharpest tool to target the cell membrane and attack on the antibodies of the human beings.

The ruptured and distorted protein envelope leads to replicate the virus at desirable conditions. However, billions and billions of replicated template copies of ssRNA will go for the considerable self-synthesis of virus and amino-acid sequence mismatching will further helps in more mutations [16]. However, Fig. 4 corroborates the passage of virus through the nasal cavity followed by the trachea and bronchus of lungs. Once virus reaches to the

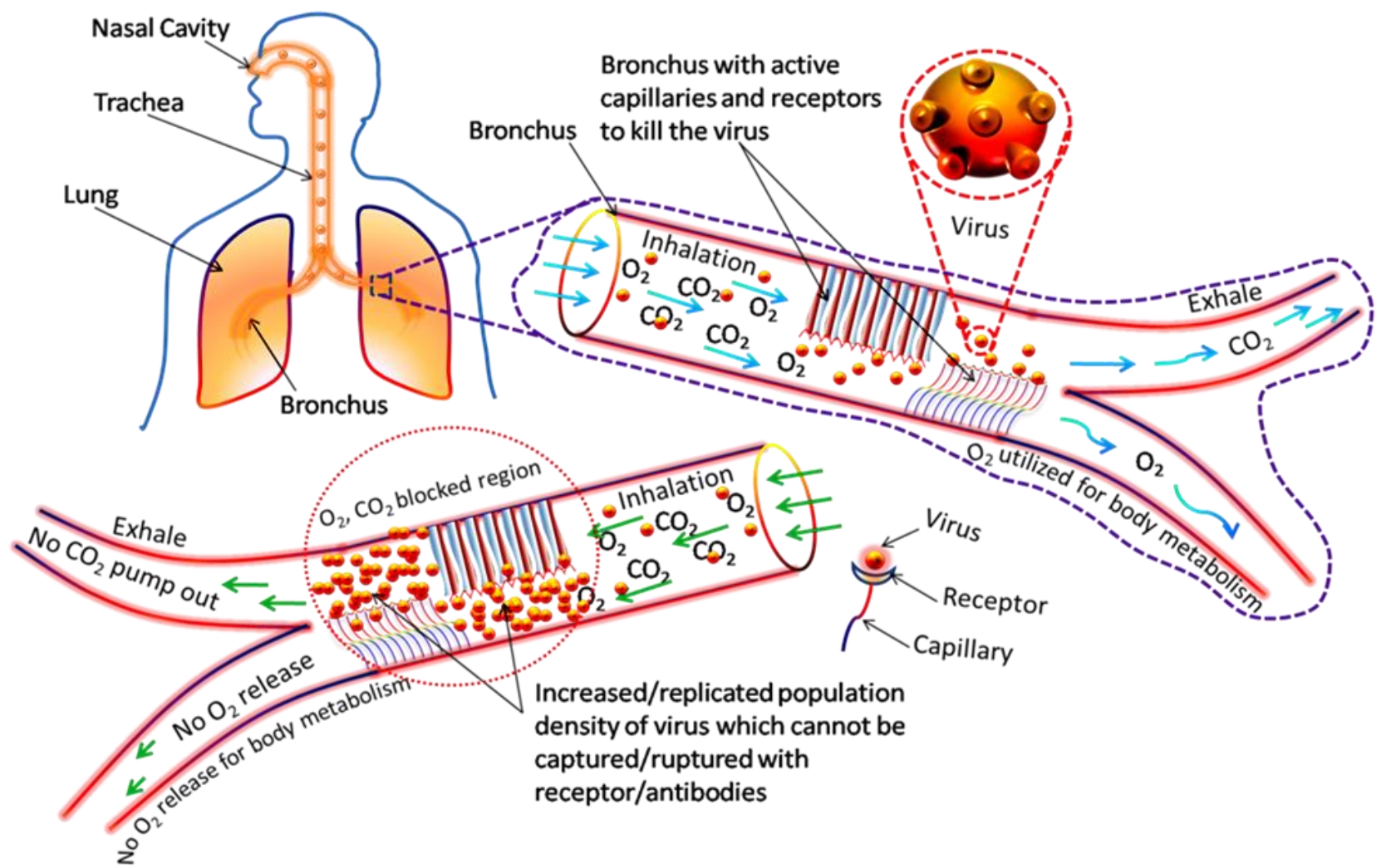

Fig. 4. Schematic shows the steps involved in the process of COVID-19 virus replication and its detrimental consequences to deactivate the receptors and blockage of oxygen and carbon dioxide gases. The complex population density of viruses inside the bronchus or capillaries lead to blockage of $\mathrm{O}_{2}$ and $\mathrm{CO}_{2}$ exchanges. 


\section{Advanced Materials Letters www.vbripress.com/aml}

bronchus, the antibody receptors which are present inside the capillaries will be activated (which means the immune system start functioning to fight with unwanted foreign agents) to rupture. The inhaled gases are the combination of many gases such as $\mathrm{O}_{2}, \mathrm{CO}_{2}, \mathrm{CO}$ (carbon monoxide) etc., and millions of capillaries which are present inside the bronchus will separate and send $\mathrm{CO}_{2}$ and $\mathrm{CO}$ for exhalation and $\mathrm{O}_{2}$ for utilization of body metabolism [17]. The inner lining of the respiratory system has been infected by causing the inflammation which leads to severe cough and blocks the gas exchange units. The air sacs which are at the bottom of the lungs will also get inflamed and end-up with pneumonia (SARS-CoV2) $[\mathbf{1 7}, \mathbf{1 8}]$. However, lungs filled with inflammatory agents (viruses) are unable to get enough $\mathrm{O}_{2}$ to supply the bloodstream for body metabolism. It reduces the capability to take the $\mathrm{O}_{2}$ and separate the $\mathrm{CO}_{2}$. This unusual phenomenon of pneumonia leads to death [18]. Ventilation and maintaining the pneumonia patient with high $\mathrm{O}_{2}$ levels until the lungs recovered is a most essential and preliminary treatment [19].

\section{Prevention methods}

Since, there is no drug or vaccine for this deadliest virus (COVID-19), till yet there is a need to take preventive methods and precautions to stay safe and healthy. Most importantly social distancing, clean environment, having warm water, frequent hand washing with soap, using the hand sanitizer, avoid the touching of the face, eyes, nose, mouth are suggestive prevention methods [20]. Moreover, stay away from touching materials, shake hands with friends and relatives, stay hygiene, bleaching the surroundings, wearing the surgical face mask is also a good practice to stay safe from virus [21]. Further avoid spitting, use the handkerchief while sneezing, frequent face washing, having neat and clean cloths, avoid unnecessary travel, using alcohol disinfectant can be an easy and most preferable prevention methods [22]. Apart from these preventive methods, self-quarantine and self-isolation is one of the foremost preventive methods from this deadliest virus to break out the chain from further spread.

\section{Conclusion}

In conclusion, the physical characteristic properties of virus such as size, shape, morphology has been elevated with proper evidences. However, the size (length) of spikes $(\sim 10$ $\mathrm{nm}$, in diameter) along with its role to lead detrimental consequences through the cell membrane penetration and net-work entanglement has been deliberated in detail. The speculations and hypothesis about virus internalization to lung cells has been explored in a mechanistic approach manner. The internal entanglement of spike spindles for network formulation and deactivation of receptor antibodies is corroborated. The virus mutations followed by the increase of population density and blockage of $\mathrm{O}_{2}$ and $\mathrm{CO}_{2}$ gas exchange has been explained. The inflammation on sacs of lungs lead to severe cough and horrific pneumonia death has been deliberated in a systemic mechanistic approach.

\section{Acknowledgments}

Authors would like to acknowledge Department of Chemistry, Zhejiang University for support and research facilities.

\section{Author's contributions}

Conceived the plan, performed the experiments, analyzed the data and wrote the paper by CA, TEM characterization and valuable inputs given by the TM, the manuscript has been corrected by the GT. Authors have no competing financial interests.

\section{Keywords}

Spikes, net-work formation, mutations, detrimental, pneumonia, inflammation.

Received: 29 May 2020

Revised: 12 July 2020

Accepted: 21 July 2020

\section{References}

1. Wu, F.; Zhao, S.; Yu, B.; Chen, Y. M.; Wang, W.; Song, Z. G.; Hu, Y.; Tao, Z. W.; Tian, T. H.; Pei, Y. Y.; Yuan, M. L.; Zhang, Y.L.; Dai, F. H.; Liu, Y.; Wang, Q. M.; Zheng, J. J.; Xu, L.; Holmes, E. C.; Zhang, Y. Z.; Nature, 2020, 579, 265.

2. Zhou, P.; Yang, X. L.; Wang, X. G.; Hu, B.; Zhang, L.; Zhang, W.; Si, H. R.; Zhu, Y.; Li, B.; Huang, C. L.; Chen, H. D.; Chen, J.; Luo, Y.; Guo, H.; Jiang, R. D.; Liu, M. Q.; Chen, Y.; Shen, X. R.; Wang, R.; Zheng, X. S.; Zhao, K.; Chen, Q. J.; Deng, F.; Liu, L. L.; Yan, B.; Zhan, F.; Wang, Y. Y.; Xiao, G. F.; Shi, Z. L.; Nature, 2020, 579, 270.

3. Li, G.; Chen, X.; Xu, A. N.; Engl. J. Med. 2003, 349, 508.

4. Meyer, B.; Drosten, C.; Muller, M. A.; Virus Res. 2014, 194, 175.

5. Zou, L.; N. Engl. J. Med., 2020, 382, 1177.

6. Li, N.; Ma, W. T.; Pang, M.; Fan, Q. L.; Hua, J. L.; Front. Immunol, 2019, 10, 1551 .

7. Singh, T.; Shukla, S.; Kumar, P.; Wahla, V.; Bajpai, V. K.; Front. Microbiol. 2017, 8, 1501.

8. Hartstra, A. V.; Bouter, K. C. E.; Backhed, F.; Nieuwdorp, M.; Diabetes Care, 2015, 38, 159.

9. Shi, Y.; Wan, Z.; Li, L.; Li, P.; Li, C.; Ma, M.; Cao, C.; J. Clin. Virol, 2004, 31, 66.

10. Lu, R.; Zhao, X.; Li, J.; Niu, P.; Yang, B.; Wu, H.; Wang, W.; Song, H.; Huang, B.; Zhu, N.; Lancet, 2019, 395, 565.

11. Huang, C.; Wang, Y.; Li, X.; Ren, L.; Zhao, J.; Hu, Y.; Zhang, L.; Fan, G.; Xu, J.; Gu, X.; Lancet, 2019, 395, 497.

12. Liu, J.; Shi, H.; Cong, G.; Chen, J.; Zhang, X.; Shi, D.; Cao, L.; Wang, X.; Zhang, J.; Ji, Z.; Jing, Z.; Feng, L.; Archives of Virology, 2020, 165, 1049.

13. Letko, M.; Marzi, A.; Munster, V.; Nature Microbiology, 2020, 5, 562.

14. Li, W.; Nature, 2003, 426, 450 .

15. Woo, P. C. Y.; Virology, 2006, 351,180.

16. Menachery, V. D.; J. Virol. 2020, 94, 01774.

17. Araya, P.; Waugh, K. A.; Sullivan, K. D.; Proc Nat Acad Sci., 2020, 116,24231

18. Zhu, N.; Zhang, D.; Wang, D.; Li, X.; Yang, B.; Song, J.; N. Engl. J. Med., 2020, 382, 727.

19. Cao, Y.; Li, L.; Feng, Z.; Wan, S.; Huang, P.; Sun, X.; Wen, F.; Huang, X.; Ning, G.; Wang, W.; Cell Discov. 2020, 6, 11.

20. Shiu, E. Y. C.; Leung, N. H. L.; .Cowling, B. J.; Curr. Opin. Infect. Dis. 2019, 32, 372.

21. Kong, W. H.; Li, Y.; Peng, M. W.; Kong, D. G.; Yang, X. B.; Wang, L.; Liu, M. Q.; Nature Microbiology, 2020, 5, 675.

22. Zou, L.; N. Engl. J. Med., 2020, 382, 1177. 